\title{
Systemic Therapy in Hepatocellular Carcinoma
}

\author{
Stephen H. Wrzesinski, MD, PhD ${ }^{a, b}$, Tamar H. Taddei, MD ${ }^{c, d}$, and Mario Strazzabosco, MD, \\ $\mathrm{PhD}^{\mathrm{c}, \mathrm{e}, \mathrm{f},{ }^{*}}$ \\ aYale Comprehensive Cancer Center, Yale University School of Medicine, New Haven, CT, USA \\ bVA Connecticut Healthcare System, Comprehensive Cancer Center, 950 Campbell Avenue- \\ 111D, West Haven, CT 06516-2700, USA \\ 'Department of Internal Medicine, Section of Digestive Diseases, Yale University School of \\ Medicine, 333 Cedar Street/1080 LMP, PO Box 208019, New Haven, CT 06520-8019, USA \\ dVA Connecticut Healthcare System, Hepatitis C Resource Center (HCRC), 950 Campbell \\ Avenue-111H, West Haven, CT 06516-2700, USA \\ eYale Liver Center, Department of Internal Medicine, Yale University, Cedar Street 333, New \\ Haven, CT 06520, USA \\ fSection of Digestive Diseases, University of Milan-Bicocca, Monza, Italy
}

\section{Keywords}

Systemic therapy; Chemotherapy; Hepatocellular carcinoma

\begin{abstract}
Hepatocellular carcinoma (HCC) is the third leading cause of cancer-related death globally. ${ }^{1}$ Other articles in this issue have addressed the epidemiology, screening, diagnosis, staging, and surgical and locoregional treatments. Although there have been many advances in HCC treatment, more than $60 \%$ of patients present with disease at a stage too advanced to benefit from curative modalities. In patients for whom surgical resection or curative ablation is an option, 50\% experience a recurrence within 18 months, and up to $70 \%$ to $80 \%$ recur within 5 years. ${ }^{2,3}$ Patients awaiting liver transplantation for HCC face the pressure of keeping their tumor downstaged, ie, downsized to stay within transplantable criteria. Thirty to forty percent of patients with HCC present with advanced disease amenable only to palliative locoregional therapies. ${ }^{4}$ Although these therapies may offer prolonged median survival, the vast majority succumb to tumor progression. ${ }^{5}$ Once advanced stage HCC has progressed through local treatment modalities, patients succumb to the disease with a median survival of about 8 months. ${ }^{6}$ The goal of treatment, therefore, becomes focused on maintaining quality of life by slowing down the progression of disease through the administration of agents with minimal side effects.
\end{abstract}

HCC is a complex cancer arising in a diseased organ. All therapies must be considered with a view to the patient's liver function and performance status. Liver dysfunction is the ratelimiting step for the key components to any therapy: tolerance, efficacy, and safety. Sequelae of cirrhosis in patients with HCC include development of portal hypertension with hypersplenism leading to platelet sequestration (potentially limiting administration of myelosuppressive agents), development of varices (with their attendant risk of bleeding),

*Corresponding author. Department of Internal Medicine, Section of Digestive Diseases, Yale University School of Medicine, 333 Cedar Street/1080 LMP, PO Box 208019, New Haven, CT 06520-8019. mario.strazzabosco@yale.edu. 
and ascites formation; hypoalbuminemia, which causes peripheral edema and may interfere with drug binding (possibly altering distribution of drugs); changes in liver vascularization and capillarization of sinusoids (leading to altered pharmacokinetics); and liver dysfunction (reducing the therapeutic margin of many drugs). Collectively, these factors make it difficult to arrive at a dose that is able to provide a therapeutic benefit with minimal side effects.

Care of the patient with HCC requires a multidisciplinary approach both clinically and scientifically. The molecular biology and genetics of hepatic oncogenesis must be considered within the context of the pathobiology of cirrhosis. The intersection of these pathways will likely provide the most promising targets and will also require the most intense innovation and collaboration among investigators.

Because advanced HCC is diagnosed in most patients, there is a great clinical need to develop effective, well-tolerated targeted systemic therapies. Thomas and colleagues ${ }^{7}$ outlined and prioritized recommendations for clinical trials research in HCC. The investigators called for trials that will identify "additional effective systemic agents, combination systemic therapies, and combined modality options." To conceive of such studies it is important to review both the failures and successes of systemic therapy for HCC and to gain an understanding of the many promising pathways for targeted therapies.

\section{OVERVIEW OF CYTOTOXIC CHEMOTHERAPY AGENTS}

There have been several cytotoxic chemotherapy regimens that have demonstrated some antitumor activity both as single agents (doxorubicin, VP-16, cisplatin, mitoxantrone, and paclitaxel) as well as in combination with other agents (Table 1). Among these agents, the most promising cytotoxic chemotherapy agent since the 1970s has been doxorubicin, initially evaluated in a study in Uganda with 14 patients and demonstrating an impressive $79 \%$ tumor response rate (RR). ${ }^{8}$ Unfortunately, subsequent larger studies could not reproduce this result, with RRs in the $10 \%$ to $20 \%$ range, comparable with what has been observed with other cytotoxic agents (see Table 1). In terms of survival benefit, the data with doxorubicin are less clear. Although a single trial randomizing 60 patients to either doxorubicin or placebo demonstrated improved median survival in the treatment group (10.6 weeks vs 7.5 weeks), ${ }^{9}$ there have been no additional studies to date demonstrating this limited survival benefit.

Although early clinical trials have suggested that combination therapies may improve RRs up to $27 \%$, overall survival (OS) has not been improved by combinations of cytotoxic chemotherapies (see Table 1). Among the combination regimens evaluated thus far, the most promising results initially came from a Phase II study assessing a regimen consisting of cisplatin (20 mg/m $/ \mathrm{m}^{2}$ intravenously [IV] Days $\left.1-4\right)$, doxorubicin (40 mg/m² IV Day 1), 5fluorouracil (5-FU) (400 mg/m $\mathrm{m}^{2}$ IV Days 1-4), and the cytokine interferon (IFN)-alpha (5 $\mathrm{MU} / \mathrm{m}^{2}$ subcutaneously, Days 1-4) (PIAF) administered every 3 weeks for up to 6 cycles. ${ }^{10}$ The regimen induced a partial response (PR) of $26 \%$ with 9 of 50 patients in this group initially with unresectable disease subsequently developing resectable disease. Among the 9 patients with resected disease, 4 were noted to have a pathologic complete response (CR), and alpha-fetoprotein (AFP) levels decreased to within acceptable limits. In spite of these encouraging initial data, the regimen was highly myelosuppressive (up to $35 \%$ with at least grade 3 leukopenia) and patients suffered from significant gastrointestinal toxicity, including at least grade 3 diarrhea (8\%) and mucositis (4\%). A subsequent Phase III study enrolling 188 patients with unresectable HCC and randomized to cisplatin/IFN alpha-2b/doxorubicin/ 5-fluorouracil (PIAF) versus single-agent doxorubicin failed to reach the study primary end point of survival benefit with median survival of 6.83 months (95\% confidence interval [CI] 
4.80-9.56) and 8.67 months (95\% CI of 6.36-12.00) for the doxorubicin arm and PIAF arm respectively $(P=.83) .{ }^{11}$

A number of factors limit the efficacy of cytotoxic agents for patients with HCC. In addition to the challenges posed by cirrhosis, the resistance of HCC to many cytotoxic agents could be attributable to the tumor itself expressing the MDR gene and drug efflux pumps such as P-glycoprotein, ${ }^{12}$ leading to efflux of the drug from the cancer cells, adding to the complexity of optimizing effective dose delivery to the tumor and its microenvironment.

Given the toxicities of multiagent chemotherapy without a reproducible clinical benefit, this approach is generally not pursued as palliative therapy in patients with advanced-stage HCC. Rather, salvage single-agent cytotoxic chemotherapies (ie, single-agent capecitabine or adriamycin) are considered after targeted therapies or clinical trial options have been exhausted.

\section{SORAFENIB INCREASES SURVIVAL IN PATIENTS WITH HCC}

In response to the lack of significant clinical benefit from cytotoxic chemotherapy, a number of agents targeting various signaling cascades in HCC (Fig. 1) have been evaluated in the Phase II setting, and Phase III trials are ongoing. After almost 3 decades of testing different cytotoxic chemotherapies for treating patients with $\mathrm{HCC}$ without demonstrating a significant clinical impact, the long-sought survival improvement was demonstrated with sorafenib.

Sorafenib is a multikinase inhibitor against the Raf1, B-Raf, vascular endothelial growth factor receptor (VEGFR)-2, platelet-derived growth factor receptor (PDGR), and c-kit pathways (see Fig. 1). The seminal Sorafenib HCC Assessment Randomized Protocol (SHARP) trial was a large, international, Phase III randomized placebo-controlled study of 602 patients with advanced HCC with 299 patients receiving sorafenib and 303 receiving placebo. ${ }^{6}$ The trial was halted following a second prespecified interim analysis because of the improvement in survival in the treatment group with the hazard ratio for sorafenib/ placebo being 0.69 (95\% CI: $0.55,0.86 ; P=.0005)$ and median survival of 10.7 months versus 7.9 months respectively. Time to progression (TTP) of disease also favored the sorafenib-treated group (5.5 months vs 2.8 months for placebo).

However, survival and TTP advantages come at a cost of a number of side effects that differ from those expected with cytotoxic chemotherapy agents. The most frequent side effects were those commonly associated with other tyrosine kinase inhibitors, including diarrhea, fatigue, hand-foot syndrome (HFS), and weight loss. Grade 3 to 4 toxicities were most common in the sorafenib arm and included diarrhea (11\% vs $2 \%$ in placebo group) and HFS ( $8 \%$ vs $1 \%$ in placebo group).

The Asia Pacific trial confirmed the survival benefit of sorafenib. ${ }^{13}$ This placebocontrolled study (150 subjects receiving sorafenib vs 76 receiving placebo) showed a similar improvement in survival and had an identical hazard ratio (OS 6.2 vs 4.1 months, $P=.014$, hazard ratio $=0.68$ ). The study population differed significantly from that in the SHARP trial, as more than $70 \%$ of patients had chronic hepatitis B, and there was a higher proportion of multifocal and metastatic disease. The inclusion of subjects with more advanced disease likely explains the lower absolute survival.

Of interest, subgroup analysis from the SHARP trial identified an even greater survival benefit for patients with hepatitis C. ${ }^{14}$ In this cohort, OS was increased to 14.0 months from 7.9 months (hazard ratio 0.58). Himmelsbach and colleagues ${ }^{15}$ have shown that sorafenib is able to interfere with $\mathrm{HCV}$ replication in vitro. This intriguing observation has yet to be validated in vivo. 
Most of the data available for sorafenib has been observed in patients with Child-TurcottePugh (CTP) A cirrhosis; further studies will be warranted to establish whether or not a true clinical benefit can be appreciated in CTP B and C patients with HCC.

The US Food and Drug Administration (FDA) has approved sorafenib for patients with unresectable HCC and, given the lack of other agents available to treat HCC, advanced CPT B patients with HCC and good Eastern Cooperative Oncology Group (ECOG) performance status (PS) are being offered sorafenib despite lack of safety and efficacy data to date. It should be emphasized that, at the present time, there is no indication for sorafenib use in patients who are candidates for ablative treatment. Studies on the use of sorafenib as an adjuvant treatment are ongoing (see the following section).

\section{SORAFENIB AS AN ADJUVANT THERAPY}

There are growing data to suggest that intrahepatic locoregional therapies may stimulate the production of cytokines, specifically VEGF, that drive tumor angiogenesis and metastasis. ${ }^{16}$ Because of sorafenib's antiangiogenic effect, several studies are under way to evaluate its efficacy in combination with locoregional therapies such as transarterial chemoembolization (TACE). The Sorafenib as Adjuvant Treatment in the Prevention of Recurrence of Hepatocellular Carcinoma (STORM) trial is a Phase III study of sorafenib as adjuvant treatment in the prevention of recurrence of hepatocellular carcinoma. The objective of this trial is to evaluate the efficacy and safety of sorafenib versus placebo in the adjuvant treatment of HCC after curative therapy (resection or ablation). The primary end point is recurrence-free survival. The study opened in 2008 and has completed recruitment (enrollment of 1065 patients). ${ }^{17}$ Completion of the study is anticipated in 2014. A Phase II study is ongoing to assess the efficacy of sorafenib after TACE, namely the Sorafenib or Placebo in Combination with Transarterial Chemoembolization (SPACE) trial for intermediate-stage hepatocellular carcinoma. This is a double-blind, placebo-controlled study that has completed recruitment (307 enrolled). The primary end point is TTP. This study is expected to be completed later this year. ${ }^{18}$ A Phase III study of TACE with or without sorafenib in unresectable HCC in patients with and without vascular invasion is actively recruiting. The purpose of the study is to assess progression-free survival (PFS). Expected enrollment is 400 patients with completion of the study anticipated in 2012. In this study, patients undergo TACE 2 weeks after a stable dose of sorafenib has been achieved. ${ }^{19}$ Data from these studies will be pivotal in clarifying the standard of care and in driving future studies with other antiangiogenic agents.

\section{SORAFENIB-BASED COMBINATION CYTOTOXIC CHEMOTHERAPY REGIMENS}

Given the relatively modest clinical benefit of single-agent sorafenib in CPT A patients with a number of toxicities of this drug, there is a clear need for further systemic therapies for patients with HCC. To this end, sorafenib-based regimens combined with cytotoxic chemotherapy (doxorubicin, capecitabine plus oxaliplatin, or gemcitabine plus cisplatin) are being evaluated. The most mature data come from a Phase II randomized control trial evaluating doxorubicin plus placebo versus doxorubicin plus sorafenib in patients with advanced HCC. Inclusion criteria included ECOG PS 0-2, CPT A cirrhosis, and no prior systemic therapies. ${ }^{20}$ Ninety-six patients were randomized to receive 3 -week cycles of doxorubicin at $60 \mathrm{mg} / \mathrm{m}^{2}$ on day 1 plus either placebo or oral sorafenib at $400 \mathrm{mg}$ twice daily for up to 6 cycles of treatment. Patients were given the option to continue with sorafenib or placebo following completion of the 6 cycles of therapy. Although the initial results were encouraging (OS and TTP were 13.7 months and 8.6 months in the doublet arm vs 6.5 months and 4.8 months in the doxorubicin arm), the potential cardiotoxicity and 
cytopenias (grades 3-4 in up to 50\% of patients in both arms) from doxorubicin raises concerns, especially when therapy in this setting is palliative.

The "control" arm was doxorubicin alone (instead of sorafenib alone); therefore, it is unclear how much, if any, benefit this specific cytotoxic agent is adding to that gained by singleagent sorafenib. Evaluation of sorafenib with other cytotoxic agents, as noted previously, will be warranted with a control arm of sorafenib alone in upcoming trials to determine whether or not combinations of this nature will lead to a clinical benefit in patients with HCC.

\section{APPROACHES OTHER THAN CYTOTOXIC CHEMOTHERAPIES OR SINGLE- AGENT SORAFENIB FOR THE TREATMENT OF HCC}

A number of targeted therapies alone and in combination with sorafenib are being evaluated, including antiestrogen/androgen agents, antiangiogenic agents, epidermal growth factor receptor (EGFR) inhibitors, mammalian target of rapamycin (mTOR) inhibitors, mitogenactivated protein (MAP) kinase kinase (MEK) inhibitors and immunotherapies with cytokines, effector cells, and antigen-presenting cells.

\section{Hormonal Manipulation}

Because HCC can express estrogen receptors and estrogens can stimulate hepatocyte proliferation in vitro and may enhance liver tumor growth in vivo, the antiestrogen, tamoxifen, has been evaluated in advanced HCC. ${ }^{21-23}$ Unfortunately, this agent did not improve survival in patients with HCC in 6 large randomized studies, 4 of which were double-blind trials. ${ }^{24}$ Furthermore, antiandrogen therapies, including flutamide or triptorelin, have also been shown to be ineffective at improving survival in patients with HCC in randomized studies. ${ }^{25}$ Somatostatin receptors are also expressed by HCC, but placebocontrolled trials of a long-acting somatostatin analog have also failed to demonstrate activity in patients with advanced $\mathrm{HCC} .{ }^{26}$

\section{Antiangiogenic Agents}

HCCs are vascular tumors with increased VEGF expression, ${ }^{27}$ and VEGF signaling cascades are proposed to induce HCC. High levels of VEGF expression correlate with worse OS in patients with $\mathrm{HCC}^{28}$; therefore, agents blocking VEGF and its downstream signaling cascade are being evaluated in this disease.

\section{Bevacizumab}

Bevacizumab, a recombinant humanized monoclonal antibody targeting VEGF, has been developed and is approved for the treatment of a number of advanced stage solid malignancies, including metastatic lung and colon cancers. ${ }^{29}$ Although this antibody does not have direct cytotoxic effects, its antitumor activity is thought to be attributable to several mechanisms, including direct antiangiogenic effects leading to decreased tumor vasculature production and "normalization" of existing tumor vasculature leading to increased interstitial pressure and possible improved delivery of cytotoxic or other targeted agents. ${ }^{30}$

Single-agent bevacizumab has been reported to demonstrate antitumor activity in 2 Phase II trials. ${ }^{31,32}$ Both trials demonstrated an RR of $12 \%$ to $13 \%$, and the trial conducted by Siegel and colleagues ${ }^{31}$ reported median PFS of 6.9 months (95\% CI, 6.5-9.1) and median survival of 12.4 months (95\% CI, 9.4-19.9) in 46 patients. It is important to note that grade 3 or higher hemorrhage occurred in $11 \%$ of patients (5/46), with 1 fatality from variceal hemorrhage. This is notable in comparison with the SHARP trial where only one subject experienced grade 3 bleeding and no grade 4 bleeding was reported. ${ }^{6}$ 
Combinations of bevacizumab with cytotoxic chemotherapies have also been evaluated in 3 Phase II studies. Of these 3, the largest Phase II study evaluated bevacizumab in combination with gemcitabine and oxaliplatin in patients with advanced HCC. ${ }^{33}$ Overall RR was reported to be $20 \%$ with an additional $27 \%$ of patients demonstrating stable disease for a median duration of 9 months. Median OS in patients receiving this combination was 9.6 months, which is less than that reported in the SHARP trial evaluating sorafenib alone; the median PFS approached that of single-agent sorafenib at 5.3 months.

In addition to the combination of bevacizumab and cytotoxic agents, a single-center Phase II trial has reported evaluating the combination of bevacizumab (10 mg/kg IV for 14 days) and the EGFR inhibitor, erlotinib ( $150 \mathrm{mg}$ by mouth daily), in patients with advanced HCC. ${ }^{34}$ The initial data are encouraging, as Thomas and colleagues ${ }^{34}$ report a $25 \%$ RR, a median PFS of 9 months, and an OS of 15 months-all outcomes that are better than that observed with single-agent sorafenib. However, similar to the results reported by Siegel and colleagues, ${ }^{31}$ in this study the rate of grade 3 or higher gastrointestinal hemorrhage was $12.5 \%$ ( $5 / 40$ patients).

Although all of the trials suggest that bevacizumab alone and in combination with cytotoxic agents and erlotinib enhanced antitumor activity, there appears to be a significantly increased risk of bleeding compared with sorafenib. Further studies with larger cohorts will be necessary to define the safety and clinical efficacy of bevacizumab alone and in combination with the agents described previously.

\section{Sunitinib}

A number of small molecule inhibitors in addition to sorafenib have been developed that block the VEGF pathway, as well as other signaling cascades. Sunitinib is an oral multikinase inhibitor targeting VEGFR-1, VEGFR-2, PDGFR-a/b, c-kit, FLt-3, and rearranged during transfection (RET) kinases and is currently approved for the treatment of advanced renal cell cancer. ${ }^{35}$ Two Phase II studies have evaluated this agent at different dosing schedules in patients with advanced HCC. The first study evaluated sunitinib administered orally at $37.5 \mathrm{mg}$ daily for 4 weeks on, 2 weeks off, and the primary end point was PFS. ${ }^{36}$ Among the 34 patients participating in this study, 1 patient had a PR lasting 20 months whereas an additional 10 patients had stable disease lasting at least 12 weeks. PFS was reported to be less than that observed with sorafenib at 3.9 months with OS approaching that of sorafenib at 9.8 months. The second Phase II study evaluated the administration of sunitinib at $50 \mathrm{mg}$ daily for 4 weeks on and 2 weeks off in patients with unresectable HCC, with the primary end point being overall RR according to the Response Evaluation Criteria in Solid Tumors (RECIST). ${ }^{37}$ Thirty-seven patients were enrolled and 1 patient also experienced a PR with 13 additional patients demonstrating stable disease. Median OS was reported to be 8.0 months and a PFS similar to the previous trial at 3.7 months.

When comparing the 2 dosages, as expected, the first study using the lower dosage of 37.5 $\mathrm{mg}$ reported lower grade 3 or 4 toxicities compared with the second study using the 50-mg higher dosage that resulted in increased grade 3 or 4 toxicities as well as a higher death rate $(10 \%)$.

Although there appears to be a biologic signal from both studies demonstrating a clinical benefit from this agent, a head-to-head randomized Phase III study evaluating sunitinib at the $37.5 \mathrm{mg}$ daily dosage versus sorafenib at $400 \mathrm{mg}$ twice daily dosing in HCC was terminated because of a higher prevalence of serious adverse effects in the sunitinib arm. ${ }^{38}$ 


\section{Brivanib}

Like sorafenib, brivanib is also a small molecular inhibitor of VEGFR-2; however, it inhibits the fibroblast growth factor receptor (FGFR) signaling pathway (see Fig. 1) as well, and has been shown to inhibit HCC growth in a mouse xenograft model. ${ }^{39}$ Recently a Phase II study was conducted to evaluate the safety and efficacy of this agent in patients with unresectable or metastatic $\mathrm{HCC}$ who either never received prior systemic treatment (cohort A) or progressed on one regimen of angiogenesis inhibitor (cohort B). ${ }^{40}$ In this study, 55 patients were in cohort $A$ and 41 patients were in cohort $B$ and all patients received oral brivanib at $800 \mathrm{mg}$ daily. The median survival of patients in cohort A was reported to be 10 months with TTP of 2.8 months with a PR observed in $5 \%$ of patients.

In both cohorts, more than $50 \%$ of patients demonstrated a greater than $40 \%$ decrease in AFP levels following initiation of this small molecule inhibitor. Of the side effects reported, the most common grade 3 or 4 events included fatigue (16\%), elevated aspartate aminotransferase levels (19\%), and hyponatremia (41\%) in cohort A, whereas the most common grade 3 or 4 events in patients in cohort B who had previously experienced one regimen of antiangiogenesis inhibitor included hypertension (7.3\%), diarrhea (4.9\%), and headache $(4.9 \%)$.

These clinical data have led to the development of Phase III studies of brivanib for first-line therapy (vs sorafenib in newly diagnosed patients with advanced HCC) and for patients who are sorafenib-refractory (compared with best supportive care for patients with advanced HCC). ${ }^{41,42}$ Accrual completion is projected for February 2013 and December 2011, respectively.

\section{AZD2171 (Cediranib)}

Cediranib is a potent inhibitor of all VEGFR tyrosine kinases, with additional activity against c-kit and PDGFR (see Fig. 1). In a Phase II study evaluating this small molecule inhibitor dosed at $45 \mathrm{mg}$ by mouth daily in patients with advanced HCC, ${ }^{43,44} 16$ of 19 patients developed grade 3 toxicities, with fatigue, hypertension, and anorexia accounting for most of these events. Additional cycles of this drug were refused by most patients who experienced grade 3 fatigue. Further studies are required to assess tolerability and safety of cediranib in patients with HCC.

\section{ABT-869}

ABT-869 is a selective inhibitor of both the VEGFR and PDGFR pathways (see Fig. 1). An interim analysis of an open-label, multicenter Phase II study of this inhibitor administered at $0.25 \mathrm{mg} / \mathrm{kg}$ daily in CPT A or once every other day in CPT B patients was recently reported. ${ }^{43,45}$ ABT-869 was administered until disease progression or intolerable toxicities developed; the primary end point is PFS at 16 weeks. Among the 34 patients in the study for whom data were available (28 with CTP A and 6 with CTP B cirrhosis), the RR was $8.7 \%$ (95\% CI, 1.1-28.0) for patients with CTP A cirrhosis, median PFS was 112 days (95\% CI 61-168) and OS was 295 days (95\% CI 182-333). The most common side effects were hypertension (41\%), fatigue (47\%), diarrhea (38\%), rash (35\%), proteinuria (24\%), nausea/ vomiting (24\%), cough, and peripheral edema (24\%). The most frequent grade 3 or 4 adverse events included hypertension (20.6\%) and fatigue (11.8\%). Unlike cediranib, the early efficacy and tolerability safety profiles of ABT-869 have encouraged additional development of this agent in patients with advanced HCC.

\section{GW786034 (Pazopanib)}

Pazopanib is a multityrosine kinase inhibitor that targets VEGFR, PDGFR, and c-kit signaling cascades (see Fig. 1). A Phase I study determining the maximum tolerated dose 
(MTD), safety, pharmacokinetics, pharmacodynamics, and antitumor effect of oral pazopanib in patients with locally unresectable or metastatic HCC was recently presented. Patients in this study were ECOG PFS 0-1, had adequate organ function, and could have received prior systemic therapy. ${ }^{46}$ The dosing schedule included escalations of $200 \mathrm{mg}$ to $800 \mathrm{mg}$ daily in a $3+3$ design. Of the 27 patients enrolled in this study, the MTD was 600 $\mathrm{mg}$ daily. There was a PR observed in 1 patient receiving $600 \mathrm{mg}$ daily and 1 patient who tolerated $800 \mathrm{mg}$ daily. Stable disease for greater than 4 months was reported in 11 patients (41\%). Median TTP at $600 \mathrm{mg}$ daily was 137.5 days (4-280 days).

\section{PTK787/ZK222584 (Valatanib)}

Valatanib targets all known VEGFR tyrosine kinases, including VEGFR/flt-1, VEGFR-2/ KDR-2, and VEGFR-3/Flt-4, as well PDGFR and c-kit signaling (see Fig. 1). Results of an open-label, multicenter Phase I study characterizing safety, tolerability, and pharmacokinetics of valatanib administered daily at oral doses ranging from 750 to $1250 \mathrm{mg}$ was reported by Koch and colleagues. ${ }^{47}$ The MTD for valatanib was $750 \mathrm{mg}$ daily. Among the 18 patients for whom efficacy data were available, 9 of 18 demonstrated stable disease with no PR or CR observed. Currently, there are no additional studies planned for this agent.

\section{Summary of Antiangiogenic Agents}

There are several antiangiogenic agents being evaluated for the treatment of advanced HCC, including a monoclonal antibody and small molecule inhibitors in addition to sorafenib that demonstrate some clinical efficacy. However, the trials evaluating these agents comprise small Phase I or II studies and, therefore, further studies enrolling larger numbers of patients and comparing these agents to the standard of care (sorafenib) are warranted. Given the large number of agents in this class of drugs relative to the number of patients available to enroll, careful consideration of the relative efficacy (ie, RRs, rates of stable of disease) versus the toxicities of each agent will be essential before initiating additional, larger Phase III trials that could take years to complete.

\section{EPIDERMAL GROWTH FACTOR-RECEPTOR INHIBITION}

The importance of EGFR/human epidermal growth factor receptor (HER)1 signaling following binding to EGFR and TGF- $a$ in hepatocarcinogenesis ${ }^{48}$ has provided the rationale for evaluating inhibitors of these pathways to treat patients with HCC. The small molecule inhibitors, erlotinib and gefitinib, as well as a chimeric monoclonal antibody against EGFR, cetuximab, have been studied as potential treatments for patients with advanced HCC.

\section{Erlotinib and Gefitinib}

Erlotinib and gefitinib are small molecule inhibitors of the phosphorylation of the tyrosine kinase associated with EGFR (see Fig. 1). Two Phase II clinical studies evaluating the safety and clinical efficacy of erlotinib at 150-mg daily dosing have demonstrated that this drug as a single agent has comparable clinical activity to the antiangiogenic agents described previously. ${ }^{49,50}$ In the Phase II study reported by Philip and colleagues, ${ }^{49} 3$ (9\%) of 38 patients with advanced HCC who received erlotinib experienced a PR; 12 (32\%) of 38 patients demonstrated PFS at 6 months with a median OS of 13 months for this group. In another Phase II study reported by Thomas and colleagues ${ }^{50}$ in which 40 patients with advanced HCC were enrolled, 17 patients (43\%) had PFS at 16 weeks and $28 \%$ patients had PFS at 24 weeks. No PR or CR was observed in the patients enrolled in this study.

An ECOG Phase II study (E1203) evaluated gefitinib, administered at $250 \mathrm{mg}$ daily, with a planned 2-stage design. ${ }^{51}$ Unfortunately, the clinical activity of this agent did not reflect that observed in the previously discussed erlotinib studies. Of 31 patients with advanced HCC 
enrolled in the first stage, 1 patient experienced a PR and 7 patients demonstrated stable disease. Median PFS was 2.8 months (95\% CI 1.5-3.9); median OS was 6.5 months (95\% CI, 4.4-8.9). Given the data and because the preestablished criteria for enrolling patients in the second stage were not met, the investigators concluded that this agent was not active in HCC.

\section{Cetuximab}

Cetuximab is a chimeric monoclonal antibody that is FDA approved for the treatment of advanced head and neck cancers and a subset (kras WT) of advanced colorectal cancers. ${ }^{52}$ Two Phase II trials have evaluated cetuximab as a single agent in CTP A and B patients with advanced HCC. ${ }^{53,54}$ Both trials reported no objective response in any of the patients participating in either study. Zhu and colleagues ${ }^{53}$ observed a median OS of 9.6 months (95\% CI of 4.3-12.1 months) and PFS of 1.4 months (95\% CI of 1.2-2.6) in the 30 patients enrolled in their study. Grünwald and colleagues ${ }^{54}$ reported that 12 of 27 patients enrolled in their Phase II study achieved stable disease; median TTP was reported to be 8 weeks.

Combinations of this biologic agent with cytotoxic chemotherapies have also been evaluated in 2 Phase II studies. The first Phase II study evaluated the combination of cetuximab administered at $400 \mathrm{mg} / \mathrm{m}^{2}$ loading dose followed by weekly administration at $250 \mathrm{mg} / \mathrm{m}^{2}$ with gemcitabine administered at $1000 \mathrm{mg} / \mathrm{m}^{2}$ on day 1 with oxaliplatin administered at 100 $\mathrm{mg} / \mathrm{m}^{2}$ on day 2 of a 14-day cycle until the development of disease progression or intolerable side effects. ${ }^{55}$ A $20 \%$ RR among the 45 patients enrolled in this study was reported with PFS and median OS being 4.7 months and 9.5 months respectively. The 1-year survival rate was $40 \%$. Gemcitabine/oxaliplatin doublet chemotherapy has reported antitumor activity; however, cetuximab did not demonstrate antitumor activity as a single agent. Therefore, the overall contribution of weekly cetuximab therapy to this combination regimen requires further elucidation.

A second Phase II study evaluating cetuximab with loading and weekly doses as described previously with the oral fluoropyrimidine, capecitabine, administered at $850 \mathrm{mg} / \mathrm{m}^{2}$ twice daily for 14 days and oxaliplatin administered at $130 \mathrm{mg} / \mathrm{m}^{2} \mathrm{IV}$ on day 1 of a 21 -day cycle has reported a RR of $10 \%$ (95\% CI of 1-33) and TTP of 4.3 months (95\% CI 2.3-5.0) in 20 of 25 enrolled patients with HCC with evaluable efficacy data. ${ }^{56}$

In summary, the efficacy of EGFR signaling inhibitors alone, and in the case of the cetuximab experience in combination with cytotoxic chemotherapy, has been modest at best. Further trials evaluating erlotinib in combination with sorafenib or bevacizumab are under way.

\section{MEK INHIBITION}

HCC frequently demonstrates MEK/extracellular signal-regulated kinase (ERK) activation. A MEK inhibitor, AZD6244, was recently evaluated in a multicenter Phase II study using RR as its primary end point in patients with advanced HCC receiving $100 \mathrm{mg}$ administered by mouth twice daily. Unfortunately, although this drug was well tolerated, there was minimal clinical efficacy observed in the treated groups with no RRs reported in the 16 patients with evaluable data. Stable disease was observed in $37.5 \%$ of patients; median TTP was a dismal 8 weeks $\left(95 \%\right.$ CI 6.6-11.1). ${ }^{57}$

\section{MTOR INHIBITION}

mTOR regulates protein translation, angiogenesis, and cell-cycle progression in many cancers including HCC, and several mTOR inhibitors are used clinically (temsirolimus, 
everolimus, and sirolimus). Furthermore, retrospective studies evaluating liver transplant patients noted that those receiving sirolimus immunosuppression had a lower prevalence of developing $\mathrm{HCC}$ when compared with those receiving calcineurin inhibitors, providing the rationale for evaluating these agents in patients with $\mathrm{HCC} .{ }^{58}$

The clinical studies evaluating this approach are in early phases. A pilot study evaluating sirolimus in 14 patients with advanced HCC reported a 40\% RR (5 patients with PR, 1 patient with CR $)^{59}$ and global studies evaluating sirolimus and everolimus are under way.

\section{SUMMARY OF "TARGETED” AGENTS IN ADDITION TO SORAFENIB}

Thus far, the results from a number of Phase II studies have yielded disappointing results with many of the targeted agents when evaluated as single agents. Many agents did not demonstrate a RR or significant improvement in survival over that observed with singleagent sorafenib. These studies have been hampered by low enrollment numbers and, given the difficulties in accruing large numbers to these types of studies, there have been too many competing studies between various agents.

It is imperative to prioritize studies evaluating agents that have strong preclinical evidence demonstrating antitumor effects in preclinical models of HCC. These drugs must be compared with sorafenib as the control arm in the first-line setting. In addition, more agents with novel mechanisms of action should be considered high priority, given the relative ineffective clinical activity of many of the older "targeted" agents. Although sorafenib has sparked a renewed hope that systemic agents can improve clinical outcomes in patients with advanced HCC, there is certainly a great need for additional approaches for the treatment of this devastating disease.

\section{IMMUNOTHERAPIES}

Given the lack of effective targeted therapies and cytotoxic therapies for advanced HCC, novel approaches evaluating the use of cytokine-based, adoptive effector cell-based, and vaccine-based immunotherapeutic approaches have been evaluated. Of interest, results of preclinical and clinical studies have demonstrated that these tumors are not classically immunogenic in mice or humans, ${ }^{60}$ warranting the use of immunotherapy to elicit antigen presentation to develop and maintain antitumor responses in the host.

\section{Cytokines}

Among the cytokines evaluated, IFN- $a$ was initially evaluated in 2 randomized trials, as interferons have immunomodulatory, antiproliferative, and antiangiogenic effects that may inhibit mechanisms critical for HCC development and progression. ${ }^{61,62}$ Unfortunately, whereas one trial performed in the 1980 s demonstrated a marginal survival advantage in patients with HCC receiving IFN-a (14.5 vs 7.5 weeks, $P=.047)$, ${ }^{61}$ a second randomized European trial did not report a survival benefit and the treatment was poorly tolerated. ${ }^{62}$ Mazzaferro and colleagues ${ }^{63}$ conducted a randomized control trial of IFN-a for prevention of HCC recurrence after liver resection in patients with hepatitis C. Although there were no differences in recurrence-free survival in the control and treatment arms, the subgroup of patients with hepatitis $\mathrm{C}$ alone (in contrast to hepatitis $\mathrm{B}$ and $\mathrm{C}$ coinfection) who were adherent to therapy had far fewer late recurrences. Further study is required to understand the potential benefit of IFN- $a$ in this group.

In addition to single-agent IFN- $\mathbf{a}$, combinations of this cytokine with cytotoxic chemotherapy agents, including PIAF, have also been evaluated, as previously discussed. A subset analysis of patients with HCC receiving the PIAF regimen who were considered 
"good-risk patients" with preserved liver function and a bilirubin level lower than $0.6 \mathrm{mg} /$ $\mathrm{dL}$, demonstrated RRs of 50\%. ${ }^{10}$ Therefore, although this regimen can be highly toxic, it may be appropriate for patients with adequate liver function in which downstaging of disease may enable subsequent surgical resection.

Several cytokine-based approaches have been studied for advanced HCC. These include TACE with IFN- $\gamma$ plus interleukin (IL)-2, ${ }^{64}$ IFN- $\gamma$ plus recombinant granulocytemacrophage colony-stimulating factor (GMCSF), ${ }^{65}$ and intratumoral injection of an adenoviral vector expressing IL-12. ${ }^{66}$ The small number of patients enrolled in these studies (up to 20 patients were enrolled in the TACE study) and the lack of significant numbers responding to the latter two approaches, suggest that further evaluation both at the bench and the bedside is required to determine the best candidate cytokines for further clinical development.

\section{Effector Cells}

The rationale for the isolation and expansion of effector cells responsible for clearing tumors in cancer patients has been well established in preclinical and clinical studies, including those for metastatic melanoma, ${ }^{67}$ suggesting that this approach may be viable for other solid tumors, such as HCC. The use of lymphocyte-activated killer (LAK) cells, transfer of ex vivo expanded tumor-infiltrating lymphocytes, and IL-2 and anti-CD3 activated peripheral blood lymphocytes have been evaluated in patients with HCC with largely disappointing and conflicting results.

These studies have been reviewed recently by Giglia and colleagues. ${ }^{66}$ In an early study, decreased rates of HCC recurrence were reported in patients who underwent resection of their primary tumor followed by infusion of LAK cells generated from peripheral blood lymphocytes obtained by leukapheresis and treated with IL-2 therapy. However, a subsequent study performed 4 years later demonstrated no clinical benefit to this approach.

The generation of tumor-infiltration lymphocyte (TIL)-based approaches has been established in patients with metastatic melanoma, resulting in excellent durable RRs. ${ }^{67}$ This approach is time and resource intensive, requiring the isolation of TILs from tumor samples and expansion of the lymphocytes ex vivo for several weeks followed by the subsequent infusion of expanded lymphocytes. Although there is one report from more than 10 years ago detailing an improved recurrence-free rate in 10 patients receiving this therapy following tumor resection, ${ }^{68}$ further studies are warranted to determine if this therapy merits further development. To date, there are no data published evaluating this approach in patients with unresectable or advanced hepatocellular carcinoma.

Finally, the use of IL-2 and anti-CD3 activated peripheral blood lymphocytes (PBLs) has been evaluated in patients with HCC by Takayama and colleagues. ${ }^{69}$ Although statistically significant improvements in the risk of recurrence and recurrence-free survival were reported, OS was not statistically significant. ${ }^{69}$

In summary, although the expansion of effector cell populations to augment antitumor responses in HCC has been evaluated, the lack of significant clinical benefit (ie, OS) and the requirement for significant resources to isolate and expand these lymphocyte populations limits further clinical development of this strategy.

\section{Use of Antigen-Presenting Cells}

A number of groups have evaluated dendritic cell (DC)-based vaccines and reported variable clinical responses. In these studies, DCs are harvested from patients with HCC, pulsed with HCC tumor antigens, and subsequently reinfused into the patient. Among the studies 
currently published, the largest one involved 31 patients treated with autologous DCs pulsed with tumor lysate. ${ }^{70}$ Four patients receiving the DC-vaccine demonstrated a PR; 17 patients had stable disease. Furthermore, 1-year survival in the 17 patients receiving monthly boosts of vaccine was reported to be $63 \%$. All treated patients demonstrated a 1-year survival rate of $40 \%$ when compared with historical controls (20\%), suggesting that the DC approach should be further evaluated in a larger cohort of patients.

In summary, immunotherapy with cytokines, effector cells, and DC-based vaccines has demonstrated limited clinical activity. The DC-based vaccines and IFN-based biochemotherapies have resulted in the largest RRs in patients with HCC. The biologic "signals" demonstrated in the trials detailed previously suggest that immunotherapeutic approaches may be a viable strategy alone and in combination with either cytotoxic chemotherapies or targeted therapies in this patient population. However, the results are limited to small clinical trials in heterogeneous populations. These approaches, developed in parallel with an improved understanding of other immunosuppressive factors in the HCC tumor microenvironment, are promising, but much work remains to be done before immunotherapy becomes standard of care for patients afflicted with this disease.

\section{SUMMARY}

Many potential systemic therapies are being investigated for the treatment of hepatocellular carcinoma. HCC is a heterogeneous disease that occurs among very heterogeneous populations. The incidence of this malignancy is rising sharply and the vast majority of patients present at advanced stages. Studies must be prioritized to be of the highest quality and yield. Although the earlier dismal results with cytotoxic chemotherapies made way for the development of locoregional therapies that provided improved overall survival, truly personalized therapy will require the selection of phenotypically similar stages of disease and populations, an understanding of the complex molecular and genetic pathways leading HCC, and a keen understanding of the pathobiology of cirrhosis. Only then will we understand how to offer a particular patient at a specific stage of disease the appropriate therapy to truly prolong survival.

Until then, treatment of HCC will continue to blend science and art, and require experience and a multidisciplinary approach. Many treatments are available, often for very similar patients. These treatments are provided by physicians working in different subspecialties and departments. In our experience, the use of multidisciplinary boards is needed to guide the best plan of therapy. Until recently, HCC treatment was restricted to surgical resection, liver transplantation, and ablative treatments. The advent of drugs able to target specific signaling pathways involved in HCC progression bear the promise to change our approach to HCC treatment. It is important to emphasize, however, that at this time (1) only sorafenib and mTOR inhibitors (the latter used to reduce post-liver transplant recurrence) have entered the clinical arena; (2) sorafenib and similar drugs are not considered first-line treatment, except in advanced, unresectable HCC; and (3) objective criteria must be devised to judge tumor response (decrease in vascularization vs decrease in mass diameter) to have objective and uniform measures of success in upcoming clinical trials.

With new drugs in the pipeline and information on the use of sorafenib as an adjuvant treatment, it is very likely that the indications for systemic treatment of HCC will significantly increase, moving from a sequential to a combination treatment strategy. As alluded to in the introduction, there are several clinical conditions that eagerly await solutions and will likely become the main indications for systemic treatment. In addition to the patient with advanced stage HCC, patients treated with surgery, ablation, or TACE may benefit from systemic treatment to reduce the recurrence of their disease and/or mitigate the 
effects of cytokines released after locoregional treatment. Patients listed for transplantation who are not amenable to ablative treatment may also benefit from systemic treatment.

Clinical trials designed to understand these questions are being performed; until the results are available, we should refrain from routinely administering systemic therapy in the absence of strong evidence. Furthermore, before embarking on costly treatments with drugs characterized by a narrow therapeutic margin, it is mandatory to consider their impact on quality of life and their place in the overall plan of care.

\section{Acknowledgments}

S.H.W. and T.H.T. have nothing to disclose; M.S. received research grants from Bayer. The support of Yale University Liver Center (NIH DK 34989) to M.S. is gratefully acknowledged.

\section{REFERENCES}

1. Bosch FX, Ribes J, Diaz M, et al. Primary liver cancer: worldwide incidence and trends. Gastroenterology. 2004; 127(5 Suppl 1):S5-S16. [PubMed: 15508102]

2. Llovet JM, Schwartz M, Mazzaferro V. Resection and liver transplantation for hepatocellular carcinoma. Semin Liver Dis. 2005; 25(2):181-200. [PubMed: 15918147]

3. Livraghi T, Meloni F, Di Stasi M, et al. Sustained complete response and complication rates after radiofrequency ablation of very early hepatocellular carcinoma in cirrhosis: is resection still the treatment of choice? Hepatology. 2008; 47(1):82-89. [PubMed: 18008357]

4. Bruix J, Llovet JM. Major achievements in hepatocellular carcinoma. Lancet. 2009; 373(9664):614616. [PubMed: 19231618]

5. Bruix J, Sala M, Llovet JM. Chemoembolization for hepatocellular carcinoma. Gastroenterology. 2004; 127(5 Suppl 1):S179-S188. [PubMed: 15508083]

6. Llovet JM, Ricci S, Mazzaferro V, et al. Sorafenib in advanced hepatocellular carcinoma. N Engl J Med. 2008; 359(4):378-390. [PubMed: 18650514]

7. Thomas MB, Jaffe D, Choti MM, et al. Hepatocellular carcinoma: consensus recommendations of the National Cancer Institute Clinical Trials Planning Meeting. J Clin Oncol. 2010; 28(25):39944005. [PubMed: 20679622]

8. Olweny CL, Toya T, Katongole-Mbidde E, et al. Treatment of hepatocellular carcinoma with adriamycin. Preliminary communication. Cancer. 1975; 36(4):1250-1257. [PubMed: 169983]

9. Lai CL, Wu PC, Chan GC, et al. Doxorubicin versus no antitumor therapy in inoperable hepatocellular carcinoma. A prospective randomized trial. Cancer. 1988; 62(3):479-483. [PubMed: 2839280]

10. Leung TW, Patt YZ, Lau WY, et al. Complete pathological remission is possible with systemic combination chemotherapy for inoperable hepatocellular carcinoma. Clin Cancer Res. 1999; 5(7): 1676-1681. [PubMed: 10430068]

11. Yeo W, Mok TS, Zee B, et al. A randomized phase III study of doxorubicin versus cisplatin/ interferon alpha-2b/doxorubicin/fluorouracil (PIAF) combination chemotherapy for unresectable hepatocellular carcinoma. J Natl Cancer Inst. 2005; 97(20):1532-1538. [PubMed: 16234567]

12. Kato A, Miyazaki M, Ambiru S, et al. Multidrug resistance gene (MDR-1) expression as a useful prognostic factor in patients with human hepatocellular carcinoma after surgical resection. J Surg Oncol. 2001; 78(2):110-115. [PubMed: 11579388]

13. Cheng AL, Kang YK, Chen Z, et al. Efficacy and safety of sorafenib in patients in the Asia-Pacific region with advanced hepatocellular carcinoma: a phase III randomised, double-blind, placebocontrolled trial. Lancet Oncol. 2009; 10(1):25-34. [PubMed: 19095497]

14. Bolondi L, Caspary W, Bennouna J, et al. Clinical benefit of sorafenib in hepatitis C patients with hepatocellular carcinoma (HCC): subgroup analysis of the sharp trial. J Clin Oncol. 2008; 26(Suppl):A129.

15. Himmelsbach K, Sauter D, Baumert TF, et al. New aspects of an anti-tumour drug: sorafenib efficiently inhibits HCV replication. Gut. 2009; 58(12):1644-1653. [PubMed: 19710032] 
16. Schoenleber SJ, Kurtz DM, Talwalkar JA, et al. Prognostic role of vascular endothelial growth factor in hepatocellular carcinoma: systematic review and metaanalysis. Br J Cancer. 2009; 100(9):1385-1392. [PubMed: 19401698]

17. [Accessed April 1, 2011] Available at: http://www.clinicaltrials.gov/ct2/show/NCT00692770

18. [Accessed April 1, 2011] Available at: http://www.clinicaltrials.gov/ct2/show/NCT00855218

19. [Accessed April 1, 2011] Available at: http://www.clinicaltrials.gov/ct2/show/NCT01004978? term5 NCT01004978

20. Abou-Alfa GK, Johnson P, Knox JJ, et al. Doxorubicin plus sorafenib vs doxorubicin alone in patients with advanced hepatocellular carcinoma: a randomized trial. JAMA. 2010; 304(19):21542160. [PubMed: 21081728]

21. Castells A, Bruix J, Bru C, et al. Treatment of hepatocellular carcinoma with tamoxifen: a doubleblind placebo-controlled trial in 120 patients. Gastroenterology. 1995; 109(3):917-922. [PubMed: 7657122]

22. Liu CL, Fan ST, Ng IO, et al. Treatment of advanced hepatocellular carcinoma with tamoxifen and the correlation with expression of hormone receptors: a prospective randomized study. Am J Gastroenterol. 2000; 95(1):218-222. [PubMed: 10638587]

23. Chow PK, Tai BC, Tan CK, et al. High-dose tamoxifen in the treatment of inoperable hepatocellular carcinoma: a multicenter randomized controlled trial. Hepatology. 2002; 36(5): 1221-1226. [PubMed: 12395333]

24. Nowak AK, Stockler MR, Chow PK, et al. Use of tamoxifen in advanced-stage hepatocellular carcinoma. A systematic review. Cancer. 2005; 103(7):1408-1414. [PubMed: 15744746]

25. Manesis EK, Giannoulis G, Zoumboulis P, et al. Treatment of hepatocellular carcinoma with combined suppression and inhibition of sex hormones: a randomized, controlled trial. Hepatology. 1995; 21(6):1535-1542. [PubMed: 7768497]

26. Becker G, Allgaier HP, Olschewski M, et al. Long-acting octreotide versus placebo for treatment of advanced HCC: a randomized controlled double-blind study. Hepatology. 2007; 45(1):9-15. [PubMed: 17187405]

27. Miura H, Miyazaki T, Kuroda M, et al. Increased expression of vascular endothelial growth factor in human hepatocellular carcinoma. J Hepatol. 1997; 27(5):854-861. [PubMed: 9382973]

28. Poon RT, Ho JW, Tong CS, et al. Prognostic significance of serum vascular endothelial growth factor and endostatin in patients with hepatocellular carcinoma. Br J Surg. 2004; 91(10):13541360. [PubMed: 15376182]

29. Jenab-Wolcott J, Giantonio BJ. Bevacizumab: current indications and future development for management of solid tumors. Expert Opin Biol Ther. 2009; 9(4):507-517. [PubMed: 19344286]

30. Jain RK. Antiangiogenic therapy for cancer: current and emerging concepts. Oncology (Williston Park). 2005; 19(4 Suppl 3):7-16. [PubMed: 15934498]

31. Siegel AB, Cohen EI, Ocean A, et al. Phase II trial evaluating the clinical and biologic effects of bevacizumab in unresectable hepatocellular carcinoma. J Clin Oncol. 2008; 26(18):2992-2998. [PubMed: 18565886]

32. Malka D, Dromain C, Farace F. Bevacizumab in patients with advanced hepatocellular carcinoma (HCC): preliminary results of a phase II study with circulating endothelial cell (CEC) monitoring. J Clin Oncol. 2007; 25(Suppl 18):4570.

33. Zhu AX, Blaszkowsky LS, Ryan DP, et al. Phase II study of gemcitabine and oxaliplatin in combination with bevacizumab in patients with advanced hepatocellular carcinoma. J Clin Oncol. 2006; 24(12):1898-1903. [PubMed: 16622265]

34. Thomas MB, Morris JS, Chadha R, et al. Phase II trial of the combination of bevacizumab and erlotinib in patients who have advanced hepatocellular carcinoma. J Clin Oncol. 2009; 27(6):843850. [PubMed: 19139433]

35. Faris JE, Michaelson MD. Targeted therapies: sunitinib versus interferon-alpha in metastatic RCC. Nat Rev Clin Oncol. 2010; 7(1):7-8. [PubMed: 20029441]

36. Zhu AX, Sahani DV, Duda DG, et al. Efficacy, safety, and potential biomarkers of sunitinib monotherapy in advanced hepatocellular carcinoma: a phase II study. J Clin Oncol. 2009; 27(18): 3027-3035. [PubMed: 19470923] 
37. Faivre S, Delbaldo C, Vera K, et al. Safety, pharmacokinetic, and antitumor activity of SU11248, a novel oral multitarget tyrosine kinase inhibitor, in patients with cancer. J Clin Oncol. 2006; 24(1): 25-35. [PubMed: 16314617]

38. [Accessed April 1, 2011] Available at: http://clinicaltrials.gov/ct2/show/NCT00699374.

39. Huynh H, Ngo VC, Fargnoli J, et al. Brivanib alaninate, a dual inhibitor of vascular endothelial growth factor receptor and fibroblast growth factor receptor tyrosine kinases, induces growth inhibition in mouse models of human hepatocellular carcinoma. Clin Cancer Res. 2008; 14(19): 6146-6153. [PubMed: 18829493]

40. Raoul J, Finn R, Kang Y, et al. An open-label phase II study of first- and second-line treatment with brivanib in patients with hepatocellular carcinoma (HCC). J Clin Oncol. 2009; 27(15S):4577.

41. [Accessed April 1, 2011] Available at: http://clinicaltrials.gov/ct2/show/NCT00858871

42. [Accessed April 1, 2011] Available at: http://clinicaltrials.gov/ct2/show/NCT00825955

43. Zhu AX. Systemic treatment of hepatocellular carcinoma: dawn of a new era? Ann Surg Oncol. 2010; 17(5):1247-1256. [PubMed: 20405329]

44. Alberts, S.; Morlan, B.; Kim, G. NCCTG phase II trial (N044J) of AZD2171 for patients with hepatocellular carcinoma (HCC): interim review of toxicity; American Society of Clinical Oncology 2007 Gastrointestinal Cancers Symposium; June 1-5, 2007; Orlando (FL). [abstract A-186]

45. Toh H, Chen P, Carr B, et al. A phase II study of ABT-869 in hepatocellular carcinoma (HCC): interim analysis. J Clin Oncol. 2009; 27(15S):4581.

46. Yau C, Chen P, Curtis C, et al. A phase I study of pazopanib in patients with advanced hepatocellular carcinoma. J Clin Oncol. 2009; 27(15S):3561.

47. Koch I, Baron A, Roberts S. Influence of hepatic dysfunction on safety, tolerability, and pharmacokinetics (PK) of PTK787/ZK 222584 in patients (pts) with unresectable hepatocellular carcinoma (HCC). J Clin Oncol. 2007; 23(Suppl):4134.

48. Whittaker S, Marais R, Zhu AX. The role of signaling pathways in the development and treatment of hepatocellular carcinoma. Oncogene. 2010; 29(36):4989-5005. [PubMed: 20639898]

49. Philip PA, Mahoney MR, Allmer C, et al. Phase II study of Erlotinib (OSI-774) in patients with advanced hepatocellular cancer. J Clin Oncol. 2005; 23(27):6657-6663. [PubMed: 16170173]

50. Thomas MB, Chadha R, Glover K, et al. Phase 2 study of erlotinib in patients with unresectable hepatocellular carcinoma. Cancer. 2007; 110(5):1059-1067. [PubMed: 17623837]

51. O’Dwyer P, Giantonio B, Levy D. Gefitinib in advanced unresectable hepatocellular carcinoma: results from the Eastern Cooperative Oncology Group's study E1203 [abstract]. J Clin Oncol. 2006; 24(18S):A-4143.

52. Markman B, Capdevila J, Elez E, et al. New trends in epidermal growth factor receptor-directed monoclonal antibodies. Immunotherapy. 2009; 1(6):965-982. [PubMed: 20635913]

53. Zhu AX, Stuart K, Blaszkowsky LS, et al. Phase 2 study of cetuximab in patients with advanced hepatocellular carcinoma. Cancer. 2007; 110(3):581-589. [PubMed: 17583545]

54. Gru nwald V, Wilkens L, Gebel M, et al. A phase II open-label study of cetuximab in unresectable hepatocellular carcinoma: final results. J Clin Oncol. 2007; 25(Suppl):4598.

55. Asnacios A, Fartoux L, Romano O, et al. Gemcitabine plus oxaliplatin (GEMOX) combined with cetuximab in patients with progressive advanced stage hepatocellular carcinoma: results of a multicenter phase 2 study. Cancer. 2008; 112(12):2733-2739. [PubMed: 18412149]

56. O’Neil B, Bernard S, Goldberg R. Phase II study of oxaliplatin, capecitabine, and cetuximab in advanced hepatocellular carcinoma. J Clin Oncol. 2008; 26(Suppl):4604.

57. O’Neil B, Williams-Goff L, Kauh J, et al. A phase II study of AZD6244 in advanced or metastatic hepatocellular carcinoma. J Clin Oncol. 2009; 27(15S):e15574.

58. Geissler EK. The impact of mTOR inhibitors on the development of malignancy. Transplant Proc. 2008; 40(Suppl 10):S32-S35. [PubMed: 19100904]

59. Decaens, T.; Luciani, A.; Itti, E. Pilot study of sirolimus in cirrhotic patients with advanced hepatocellular carcinoma; Gastrointestinal Cancers Symposium; January 15-17; San Francisco (CA). 2009. [abstract 244] 
60. Overwijk WW, Theoret MR, Finkelstein SE, et al. Tumor regression and autoimmunity after reversal of a functionally tolerant state of self-reactive CD81 T cells. J Exp Med. 2003; 198(4): 569-580. [PubMed: 12925674]

61. Lai CL, Lau JY, Wu PC, et al. Recombinant interferon-alpha in inoperable hepatocellular carcinoma: a randomized controlled trial. Hepatology. 1993; 17(3):389-394. [PubMed: 8383088]

62. Llovet JM, Sala M, Castells L, et al. Randomized controlled trial of interferon treatment for advanced hepatocellular carcinoma. Hepatology. 2000; 31(1):54-58. [PubMed: 10613728]

63. Mazzaferro V, Romito R, Schiavo M, et al. Prevention of hepatocellular carcinoma recurrence with alpha-interferon after liver resection in HCV cirrhosis. Hepatology. 2006; 44(6):1543-1554. [PubMed: 17133492]

64. Lygidakis NJ, Kosmidis P, Ziras N, et al. Combined transarterial targeting locoregional immunotherapy-chemotherapy for patients with unresectable hepatocellular carcinoma: a new alternative for an old problem. J Interferon Cytokine Res. 1995; 15(5):467-472. [PubMed: 7544232]

65. Reinisch W, Holub M, Katz A, et al. Prospective pilot study of recombinant granulocytemacrophage colony-stimulating factor and interferon-gamma in patients with inoperable hepatocellular carcinoma. J Immunother. 2002; 25(6):489-499. [PubMed: 12439346]

66. Giglia JL, Antonia SJ, Berk LB, et al. Systemic therapy for advanced hepatocellular carcinoma: past, present, and future. Cancer Control. 2010; 17(2):120-129. [PubMed: 20404795]

67. Rosenberg SA, Dudley ME. Adoptive cell therapy for the treatment of patients with metastatic melanoma. Curr Opin Immunol. 2009; 21(2):233-240. [PubMed: 19304471]

68. Wang Y, Chen $\mathrm{H}, \mathrm{Wu} \mathrm{M}$, et al. Postoperative immunotherapy for patients with hepatocarcinoma using tumor-infiltrating lymphocytes. Chin Med J (Engl). 1997; 110(2):114-117. [PubMed: 9594281]

69. Takayama T, Sekine T, Makuuchi M, et al. Adoptive immunotherapy to lower post-surgical recurrence rates of hepatocellular carcinoma: a randomised trial. Lancet. 2000; 356(9232):802807. [PubMed: 11022927]

70. Lee WC, Wang HC, Hung CF, et al. Vaccination of advanced hepatocellular carcinoma patients with tumor lysate-pulsed dendritic cells: a clinical trial. J Immunother. 2005; 28(5):496-504. [PubMed: 16113606]

71. Chlebowski RT, Brzechwa-Adjukiewicz A, Cowden A, et al. Doxorubicin $(75 \mathrm{mg} / \mathrm{m} 2)$ for hepatocellular carcinoma: clinical and pharmacokinetic results. Cancer Treat Rep. 1984; 68(3): 487-491. [PubMed: 6322986]

72. Melia WM, Johnson PJ, Williams R. Induction of remission in hepatocellular carcinoma. A comparison of VP 16 with adriamycin. Cancer. 1983; 51(2):206-210. [PubMed: 6295596]

73. Falkson G, Ryan LM, Johnson LA, et al. A random phase II study of mitoxantrone and cisplatin in patients with hepatocellular carcinoma. An ECOG study. Cancer. 1987; 60(9):2141-2145. [PubMed: 2830952]

74. Colleoni M, Buzzoni R, Bajetta E, et al. A phase II study of mitoxantrone combined with betainterferon in unresectable hepatocellular carcinoma. Cancer. 1993; 72(11):3196-3201. [PubMed: 8242542]

75. Chao Y, Chan WK, Birkhofer MJ, et al. Phase II and pharmacokinetic study of paclitaxel therapy for unresectable hepatocellular carcinoma patients. Br J Cancer. 1998; 78(1):34-39. [PubMed: 9662247]

76. O'Reilly EM, Stuart KE, Sanz-Altamira PM, et al. A phase II study of irinotecan in patients with advanced hepatocellular carcinoma. Cancer. 2001; 91(1):101-105. [PubMed: 11148565]

77. Fuchs CS, Clark JW, Ryan DP, et al. A phase II trial of gemcitabine in patients with advanced hepatocellular carcinoma. Cancer. 2002; 94(12):3186-3191. [PubMed: 12115351]

78. Patt YZ, Hassan MM, Aguayo A, et al. Oral capecitabine for the treatment of hepatocellular carcinoma, cholangiocarcinoma, and gallbladder carcinoma. Cancer. 2004; 101(3):578-586. [PubMed: 15274071]

79. Gish RG, Porta C, Lazar L, et al. Phase III randomized controlled trial comparing the survival of patients with unresectable hepatocellular carcinoma treated with nolatrexed or doxorubicin. J Clin Oncol. 2007; 25(21):3069-3075. [PubMed: 17634485] 
80. Louafi S, Boige V, Ducreux M, et al. Gemcitabine plus oxaliplatin (GEMOX) in patients with advanced hepatocellular carcinoma (HCC): results of a phase II study. Cancer. 2007; 109(7):13841390. [PubMed: 17330837]

81. Boige V, Raoul JL, Pignon JP, et al. Multicentre phase II trial of capecitabine plus oxaliplatin (XELOX) in patients with advanced hepatocellular carcinoma: FFCD 03-03 trial. Br J Cancer. 2007; 97(7):862-867. [PubMed: 17876335]

82. Shim JH, Park JW, Nam BH, et al. Efficacy of combination chemotherapy with capecitabine plus cisplatin in patients with unresectable hepatocellular carcinoma. Cancer Chemother Pharmacol. 2009; 63(3):459-467. [PubMed: 18437384]

83. Ikeda M, Okusaka T, Ueno H, et al. A phase II trial of continuous infusion of 5-fluorouracil, mitoxantrone, and cisplatin for metastatic hepatocellular carcinoma. Cancer. 2005; 103(4):756762. [PubMed: 15637692] 

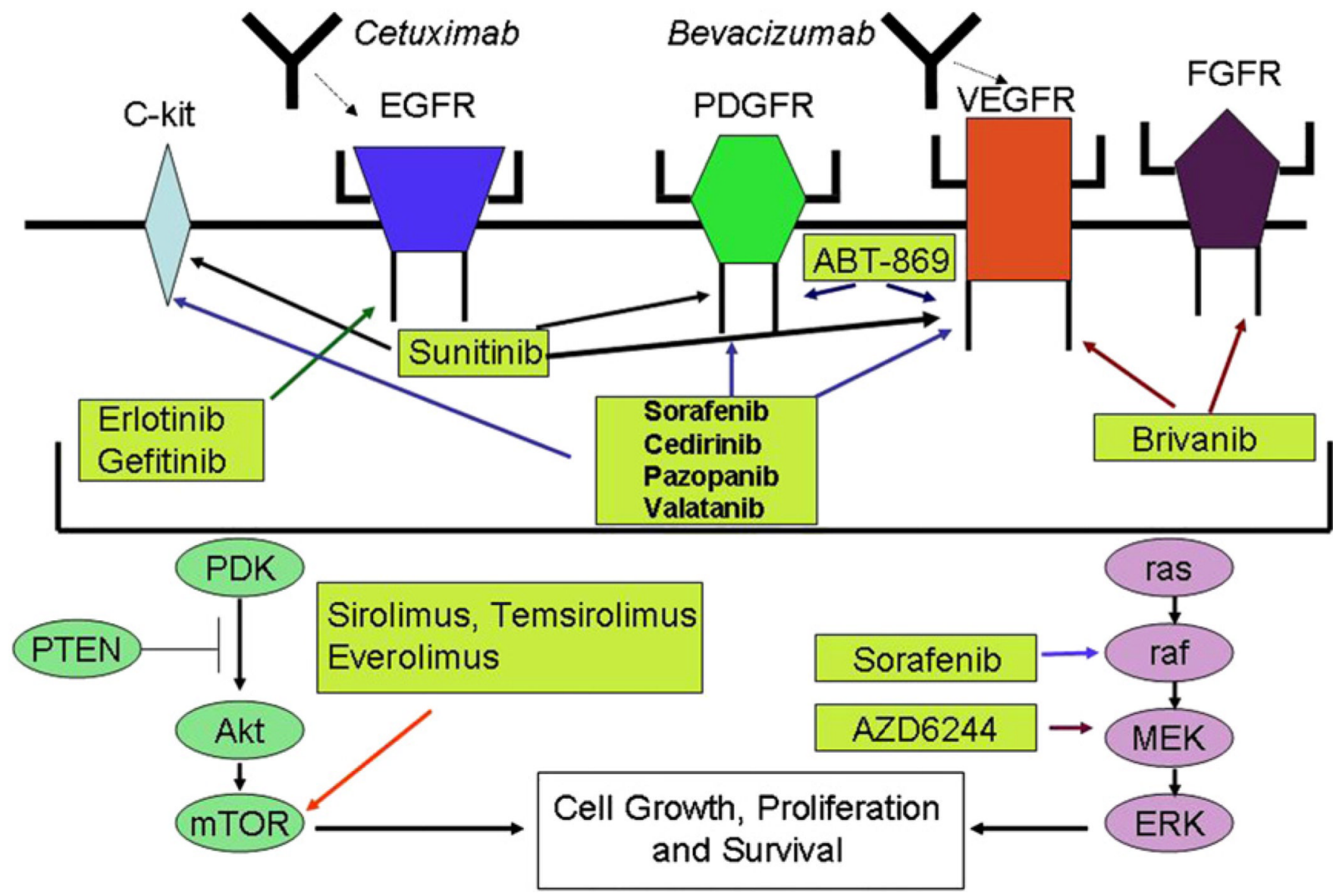

Fig. 1.

Summary of converging molecular pathways in HCC inhibited by targeted agents. Antibodies listed in italics and small molecular inhibitors in shaded rectangles. EGFR, epidermal growth factor receptor; PDGFR, platelet-derived growth factor receptor; VEGFR, vascular endothelial growth factor receptor; FGFR, fibroblast growth factor receptor; PDK, phosphate-dependent kinase; PTEN, phosphatase and tensin homolog deleted in chromosomes 10; mTOR, mammalian target of rapamycin; MEK, mitogen-activated protein kinase kinase; ERK, extracellular related kinase. 
Table 1

Summary of cytotoxic chemotherapies evaluated for HCC

\begin{tabular}{|c|c|c|}
\hline Drug & $\begin{array}{l}\text { Latest Stage of } \\
\text { Clinical Development }\end{array}$ & $\begin{array}{l}\text { Referenced Summary } \\
\text { of Results }\end{array}$ \\
\hline Doxorubicin & Phase III & RR up to $15 \%$; Median OS $6.8 \mathrm{mo}^{11,71}$ \\
\hline VP-16/Etoposide & Phase II & RR up to $18 \%$; Median OS $6.8 \mathrm{mo}^{72}$ \\
\hline Cisplatin & Phase II & RR up to $17 \%$; OS unavailable ${ }^{73}$ \\
\hline Mitoxantrone & Phase II & RR 8\%; Median OS 14 wk $^{73}$ \\
\hline Mitoxantrone $+\beta$-IFN & Phase II & RR up to $23 \%$; Median OS $8 \mathrm{mo}^{74}$ \\
\hline Paclitaxel & Phase II & RR 0\%; Median OS 12 wk $^{75}$ \\
\hline Irinotecan & Phase II & $\mathrm{RR}$ up to $7 \%^{76}$ \\
\hline Gemcitabine & Phase II & RR 0\%; Median OS $6.9 \mathrm{mo}^{77}$ \\
\hline Capecitabine & Retrospective study & RR up to $11 \%$; Median OS $10.1 \mathrm{mo}^{78}$ \\
\hline Nolatrexed & Phase III & RR up to $1.5 \%$; Median OS 22.3 wk $^{79}$ \\
\hline $\begin{array}{l}\text { Cisplatin, IFN-a, doxorubicin, and 5- } \\
\text { fluorouracil }\end{array}$ & Phase II and III & $\begin{array}{l}\text { Phase II } \\
\text { RR 26\%; Median OS } 8.9 \mathrm{mo}^{10} \\
\text { Phase III } \\
\text { RR } 21 \% \text { OS } 8.67 \text { mo PIAF arm vs OS } 6.83 \text { mo in single-agent doxorubicin } \\
\text { arm }^{11}\end{array}$ \\
\hline Gemcitabine + Oxaliplatin & Phase II & RR up to $18 \% ;$ Median OS $11.5 \mathrm{mo}^{80}$ \\
\hline Capecitabine + Oxaliplatin & Phase II & RR up to $6 \%$; Median OS $9.3 \mathrm{mo}^{81}$ \\
\hline Capecitabine + Cisplatin & Cohort & RR up to $20 \%$; Median OS $10.5 \mathrm{mo}^{82}$ \\
\hline $\begin{array}{l}\text { Cisplatin, mitoxantrone, and 5- } \\
\text { fluorouracil }\end{array}$ & Phase II & RR up to $27 \%$; Median OS $11.6 \mathrm{mo}^{83}$ \\
\hline $\begin{array}{l}\text { Gemcitabine, oxaliplatin, and } \\
\text { bevacizumab }\end{array}$ & Phase II & RR up to $20 \%$; Median OS $9.6 \mathrm{mo}^{33}$ \\
\hline
\end{tabular}

Abbreviations: IFN, interferon; OS, overall survival; RR, response rate. 